\title{
Co-existent facial palsy and myocarditis in a 50-year old farmer diagnosed with probable leptospirosis: a case report
}

\author{
Kulatunga Wijekoon Mudiyanselage Pramitha Prabhashini Kumarihamy', \\ Dissanayake Mudiyanselage Priyantha Udaya Kumara Ralapanawa ${ }^{2^{*}}$ \\ and Widana Arachchilage Thilak Ananda Jayalath ${ }^{2}$
}

\begin{abstract}
Background: Leptospirosis is a worldwide zoonotic disease caused by spirochetes belonging to the genus Leptospira. This is a case report on a patient with probable leptospirosis, who developed lower motor neuron facial nerve palsy during the recovery phase of this illness. Leptospirosis is endemic in Sri Lanka and this complication has been reported earlier in other countries but not in Sri Lanka to the best of our knowledge.

Case presentation: A previously well 50 year old Sinhalese farmer in Sri Lanka was admitted to a tertiary care hospital with five day history of fever, headache, prostration, severe myalgia especially in the calves and yellowish discoloration of the eyes. He was febrile, icteric and had suffusion of both conjunctivae. His pulse rate and blood pressure was $98 / \mathrm{min}$ and $90 / 50 \mathrm{mmHg}$ respectively. The initial laboratory examinations showed neutrophil leukocytosis and thrombocytopenia. Antibodies test for leptospirosis was done and IgM was positive. Because of this evidence the probable diagnosis of leptospirosis was made and antibiotic therapy initialed with intravenous cefotaxime $1 \mathrm{~g} 8$ hrly and crystalline penicillin 2 mu 6 hrly.

On the eighth day he developed chest pain associated with shortness of breath with a heart rate of 120/min. Electrocardiograpic and echocardiograpic evidence suggested myocarditis, and trponin I titer was positive.

Supportive care was provided and antibiotics were continued.

On the $13^{\text {th }}$ day of illness he developed lower motor type facial nerve palsy of the left side with positive Bell's phenomenon. But rest of the neurological examination was normal. He was discharged on a step-down course of prednisolone and physiotherapy. He fully recovered from cardiac involvement before discharge but recovery from facial nerve palsy took place only six months later.

Conclusion: Our case emphasizes cardiac and facial nerve involvement in leptospirosis. This is the first published report in Sri Lanka of facial nerve palsy occurring in leptospirosis possibly due to immunological damage. Further literature survey revealed that this is the first case in the world with simultaneous occurrence of myocarditis and facial nerve palsy in leptospirosis. The pathogenesis of this occurrence is yet to be fully understood.
\end{abstract}

Keywords: Leptospirosis, Myocarditis, Facial nerve palsy, Sri Lanka

\footnotetext{
* Correspondence: udayapralapanawa@yahoo.com

${ }^{2}$ Department of Medicine, University of Peradeniiya, Peradeniiya, Sri Lanka

Full list of author information is available at the end of the article
} 


\section{Background}

Leptospirosis is a re-emerging zoonotic disease of worldwide occurrence caused by the spirochetes belonging to the genus Leptospira. [1,2]. It is endemic in the tropical and subtropical region [1] and characterized by acute febrile illness with mild self-limiting infection or a more severe, and often fatal, multiorgan involvement including renal failure and myocarditis [1]. This is a case report of a patient with probable leptospirosis, who developed lower motor neuron facial nerve palsy during the recovery phase of this illness. This complication of leptospirosis has been reported earlier [3-5] in other countries but not in Sri Lanka to the best of our knowledge even though it is endemic to our country [6].

\section{Case presentation}

A 50 year old previously well Sri Lankan Sinhalese farmer was admitted on $17^{\text {th }}$ June 2013 , to a tertiary care hospital with five day history of fever. He developed high grade fever, headache, prostration and severe myalgia especially in the calves that necessitated the need for hospitalization. He had noticed yellowish discoloration of the eyes on the day of admission to hospital and said he had been working in the faddy field for the last two weeks until he developed fever.

On clinical examination, he was febrile, icteric and had suffusion of both conjunctivae. The pulse rate was 98/min, regular and blood pressure was 90/50 $\mathrm{mmHg}$ with a respiratory rate of $30 / \mathrm{min}$. There was no hepatospleenomegaly while gentle palpation of the abdomen and calves caused considerable pain. There were no signs of meningeal irritation.

The initial laboratory examinations showed neutrophil leukocytosis (15,310 leukocytes per microliter and $85 \%$ of neutrophils) and thrombocytopenia (51,000 platelets per microliter). Serum creatinine was $325 \mathrm{UMol} / \mathrm{l}$ and urea $25.14 \mathrm{mmol} / \mathrm{L}$, with serum potassium of $4.7 \mathrm{mmol} / \mathrm{L}$. Arterial blood gas analysis showed compensated metabolic acidosis with $\mathrm{pH}$ of 7.32, bicarbonate (HCO3) of $13.9 \mathrm{mmol} / \mathrm{l}$, partial pressure of carbon dioxide $(\mathrm{pCO} 2)$ $24 \mathrm{mmol} / \mathrm{l}$. Initial urine output was low. With fluid resuscitation his urine output and renal functions improved.

Blood aspartate aminotransferase (AST) and alanine aminotransferese (ALT) were mildly elevated. Total bilirubin was $108.78 \mu \mathrm{mol} / \mathrm{L}$ with elevated direct fraction (88.43 $\mu \mathrm{mol} / \mathrm{L})$. Alkaline phosphatase was $384 \mathrm{U} / \mathrm{l}$. Urinalysis showed red blood cells. Creatinine phosphokinase (CPK) was $255 \mathrm{U} / \mathrm{L}$ and erythrocyte sedimentation rate (ESR) $85 \mathrm{~mm} / \mathrm{h}$ with $\mathrm{C}$-reactive protein (CRP) of $196 \mathrm{nmol} / \mathrm{l}$. Ultrasonography of abdomen was normal. A lumbar puncture was not performed as the patient had persistent thrombocytopenia. Leptospirosis antibodies were done and IgM [by enzyme-linked immunosorbent assay (ELISA) method] was positive.
Because of the clinical and laboratorial findings, the probable diagnosis of leptospirosis was made and the patient was transferred to the high dependency unit (HDU) and monitored regularly. Supportive care was initiated, with emphasis on the fluid resuscitation to improve renal function. Antibiotic therapy was started with intra venous cefotaxime $1 \mathrm{~g} 8 \mathrm{hrly}$ and crystalline penicillin 2 mu 6 hrly. On the third day of admission (eighth day of illness), he developed chest pain associated with shortness of breath with a heart rate of $120 / \mathrm{min}$ and electrocardiogram (ECG) showed sinus tachycardia with widespread mild ST depression. But there were no progressive ECG changes on the repeat ECGs. Troponin I was positive with high titer. The echocardiograme showed global hypokinesia with an ejection fraction of $38 \%$ with no pericardial effusion. In the light of this evidence, the diagnosis of myocarditis complicating leptospirosis was made. He was given supportive care with the continuation of antibiotics. On the $13^{\text {th }}$ day of the illness when hospital discharge was being considered due to the significant improvement of his clinical and laboratory parameters, he developed lower motor type facial nerve palsy of the left side with positive Bell's phenomenon (Figure 1). Other cranial nerves and both upper and lower limbs were neurologically normal. No clinical evidence of Herpes Zoster was found. The venereal disease research laboratory (VDRL) and human immunodeficiency virus (HIV) tests were negative. Both IgM \& IgG antibodies for varicella were negative. The nerve conduction study demonstrated axonal neuropathy of the left facial nerve. Prednisolone $40 \mathrm{mg}$ daily was initiated and terminated gradually after ten days. On discharge echocardiography (done ten days after the first echocardiography) was normal with ejection fraction of $68 \%$ without regional wall motion abnormalities.

The patient was discharged on the thirteen day of admission. He showed partial improvement of facial paralysis and was discharged on a step-down course of prednisolone. He was seen at intervals of two weeks and two months after discharge at which time there was gradual recovery from facial palsy. By six months it had improved totally (Figure 2).

\section{Discussion}

Leptospirosis is caused by the spirochetes belonging to genus leptospira [1]. This is a zoonotic disease which can cause multi-system involvement $[1,6,7]$. The pathogenesis of this organ dysfunction is not yet fully understood.

Our patient had exposure history with clinical symptoms and signs strongly consistent with leptospirosis with positive IgM antibodies by enzyme-linked immunosorbent assay (ELISA). Therefore this can be considered a probable case of leptospirosis according to case definition [8-10]. 


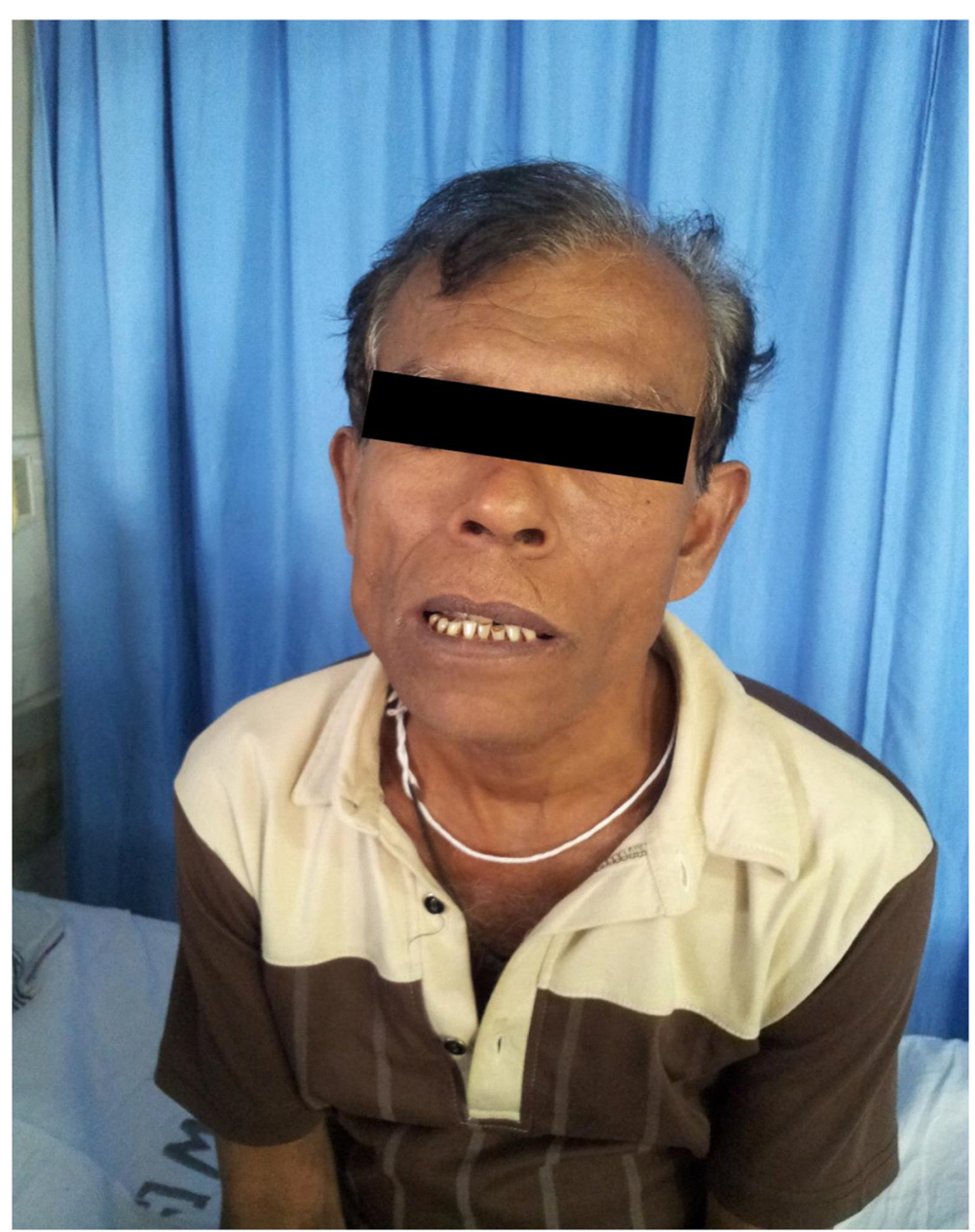

Figure 1 Shows involvement of the left frontalis muscle, mouth deviation to right side indicating left lower motor neuron type of facial nerve palsy.

Myocarditis is one of the known cardiac complications of leptospirosis infection [11,12]. But myocarditis is an underestimated complication of leptospirosis due to the fact that it is frequently asymptomatic. Leptospirosis myocarditis can have a severe evolution and at times prove even fatal [13]. During the leptospirosis outbreak in 2008 in Sri Lanka, myocarditis was reported in $7.1 \%$ of patients and heart failure in 3.9\% [13]. Navinan et al have shown that myocardial inflammation and vasculitis as the main postmortem findings of leptospirosis. Further they stated that more studies are needed to elucidate the pathophysiology of myocarditis in leptospirosis [13]. Ciuchi-Nicolau has shown that myocarditis has developed on the tenth day of illness of the leptospirosis patient [14]. Our patient developed myocarditis on the eight day of illness. By this time leptospiraemia and the body immune response might have been contributed to the development of myocarditis.
In our case myocarditis was diagnosed with clinical evidence of tachycardia, wide spread ST segment changes and sinus tachycardia on the ECG and high troponin I titre with 2D echocardiographic evidence of global hypokinesia with ejection fraction of $38 \%$ with no pericardial effusion. This was further supported by normalization of ECG and 2D echocardiographic finding within ten days with supportive care. Even though we wanted to rule out coronary artery disease via a coronary angiogram, it was not performed as our patient had recovered fully with supportive care and treatment for leptospirosis.

The occurrence of facial palsy in leptospirosis has been reported earlier in other parts of the world [3-5]. Widespread damage to muscles, nerves and the myoneuronal junctions has also been reported [15]. Although the leptospirosis is an endemic disease in Sri Lanka [6], we could not find any case report with similar association in 


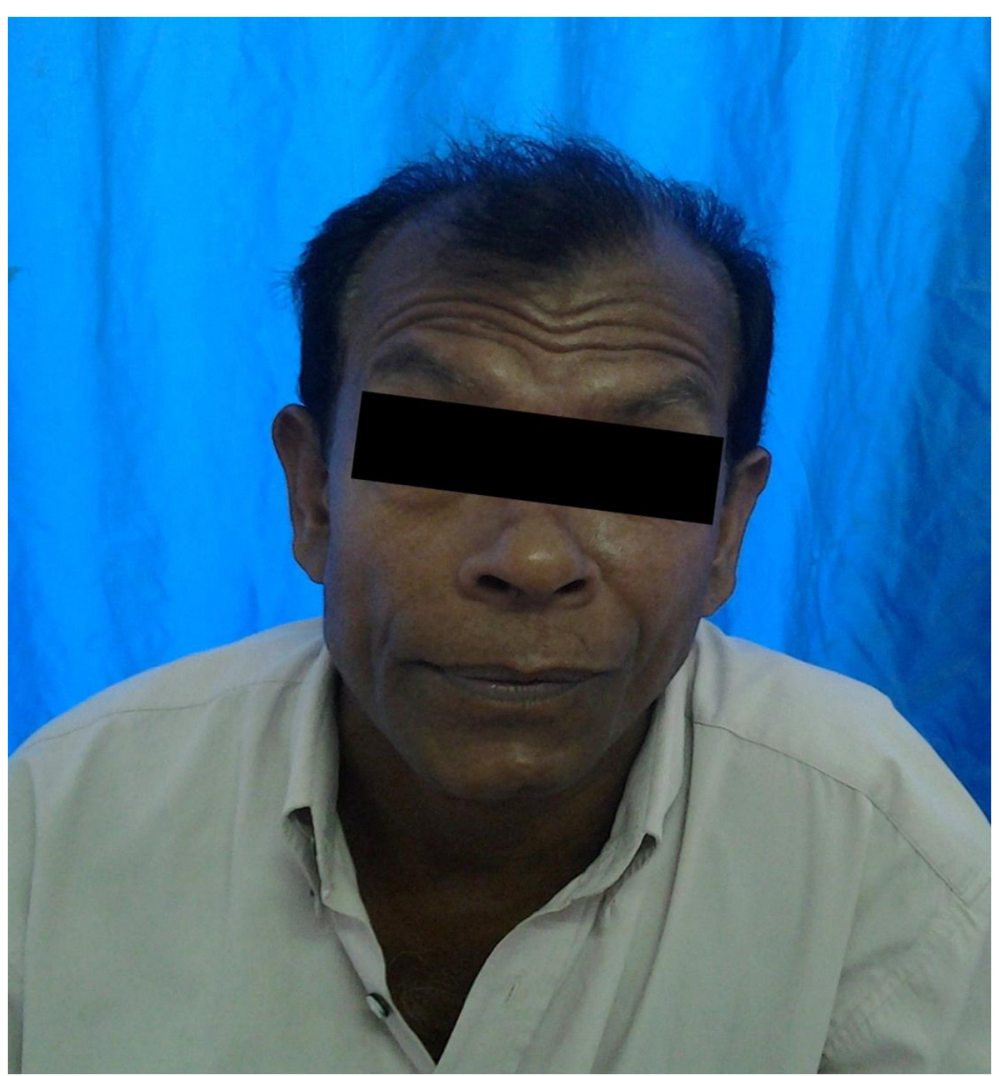

Figure 2 Total improvement of left side facial nerve palsy after 6 months.

our literature survey and presume it's the first reported case of facial palsy occurring with association of myocarditis in our country. This complication in our patient occurred in the convalescent phase of the disease as in previously reported cases [3-5]. Costa E et al shown that leptospirosis patients developed facial nerve palsy approximately on the ninth day of symptomatic disease, when the clinical manifestations attributed to leptospirosis had subsided [16]. This was also consistent with the idea that facial palsy in patients with infectious disease is mediated by an immunological mechanism [16,17] which is yet to be understood. However, it would be difficult to rule out a chance coincidence of these two pathologies.

As discussed above we could postulate that the simultaneous occurrence of myocarditis and facial nerve palsy in our patient possibly has an immunological basis. But this needs further evaluation.

\section{Conclusion}

Our case emphasizes cardiac and facial nerve involvement in leptospirosis. It is the first published such a case report in Sri Lanka on leptospirosis possibly due to immunological damage. Further literature survey revealed that this is the first case in the world with the simultaneous occurrence of myocarditis and facial nerve palsy in leptospirosis. The pathogenesis of these occurrences is yet to be fully understood.

\section{Consent}

Written informed consent was obtained from the patient for publication of this case report and any accompanying images. A copy of the written consent is available for review by the Editor-in-Chief of this journal.

\section{Abbreviations}

HCO3: Bicarbonate; PCO2: Partial pressure of carbon dioxide; AST: Aspartate aminotransferase; ALT: Alanine aminotransferese; CPK: Creatinine phosphokinase; CRP: C reactive protein; ESR: Erythrocyte sedimentation rate; ELISA enzyme: Linked immunosorbent assay; ECG: Electrocardiogram; VDRL: Venereal disease research laboratory; HIV: Human immunodeficiency virus.

\section{Competing interests}

The authors declare that they have no competing interests.

\section{Authors' contributions}

Analysis and interpretation of patient data and literature review were done by KWMPPK, DMPUKR. DMPUKR, WATAJ guided the other authors in reporting this case and corrected the final manuscript. All authors were involved in the management of the patient and read and approved the final manuscript. 
Authors' information

KWMPP (MBBS) is a registrar in medicine. DMPUKR (MBBS, MD, MRCP (UK), FRCP (London)). WATAJ (MBBS, MD, MRCP (UK), FRCP (London)) are senior Lecturer \& senior consultant physicians.

\section{Acknowledgments}

We all express our gratitude to Dr. T. Varagunam for his critical reading and grammar check of the manuscript, and to the patient, who kindly gave consent for his case to be presented in this paper.

\section{Author details}

${ }^{1}$ University Medical Unit, Teaching Hospital, Peradeniya, Sri Lanka.

${ }^{2}$ Department of Medicine, University of Peradeniiya, Peradeniiya, Sri Lanka.

Received: 13 August 2014 Accepted: 23 January 2015

Published online: 04 February 2015

\section{References}

1. Hartskeerl RA, Collares-Pereira M, Ellis WA. Emergence, control and re-emerging leptospirosis: dynamics of infection in the changing world. Clin Microbiol Infect. 2011;17:494-501.

2. Levett PN. Leptospirosis. In clinical microbiology reviews. Am Soc Microbiol. 2001;14(2):296-326.

3. El Bouazzaoui A, Houari N, Arika A, Belhoucine I, Boukatta B, Sbai H, et al. Facial palsy associated with leptospirosis. Eur Ann Otorhinolaryngol Head Neck Dis. 2011;128(5):275-7.

4. Silva AA, Ducroquet M, Junior JCP. Bilateral facial palsy associated with leptospirosis. Braz J Infect Dis. 2009;13(4):319-21.

5. Garcia-Monco JC, Benach JL. A disconnection between the neurospirochetoses in human and rodent models of disease. PLoS Pathog. 2013;9(4):e1003288

6. Agampodi S, Peacock SJ, Thevanesam V. The potential emergence of leptospirosis in Sri Lanka. Lancet Infect Dis. 2009;9(9):524-6.

7. Bharti AR, Nally JE, Ricaldi JN, Matthias MA, Diaz MM, Lovett MA, et al. Leptospirosis: a zoonotic disease of global importance. Lancet Infect Dis. 2003;3:757-71

8. Agampodi S. Case definitions in Leptospirosis: a note to Sri Lankan researchers. Sri Lankan J Infect Dis. 2012;2(2):55-7.

9. World Health Organization. Report of the Second Meeting of Leptospirosis Burden Epidemiology Reference Group. Geneva: World Health Organization; 2011. p. 9-10.

10. Okali N, Koizumi N, Kularatne SAM, Rajapakse J, Gamage CD, Muto M, et al. Leptospira infection at the University of Peradeniya Teaching Hospital, Sri Lanka: clinical and laboratory investigations. Southeast Asian J Tropmed PublicHealth. 2012;43(4):943-50

11. Abqueguen P, Delbos V, Blanvillain J, Chennebault JM, Cottin J, Fanello S, et al. Clinical aspects and prognostic factors of leptospirosis in adults. Retrospective study in France. J Infect. 2008:57:171-8.

12. Ranawaka N, Jeevagan $V$, Karunanayake $P$, Jayasinghe S. Pancreatitis and myocarditis followed by pulmonary hemorrhage, a rare presentation of leptospirosis- A case report and literature survey. BMC Infect Dis. 2013;13:38.

13. Navinan MR, Rajapakse S. Cardiac involvement in leptospirosis. Trans R Soc Trop Med Hyg. 2012;106(9):515-20.

14. Iuna Elena Ciuchi-Nicolau: Acute myocarditis due to Leptospira Icterohaemorrhagiae-A case report. Ther Pharmacol Clin Toxicol 2010 XIV(2):140-45

15. Pradhan S, Tandon R, Kishore J. Combined involvement of muscle, nerve, and myoneural junction following leptospira infection. Nurol India. 2012;60(5):514-6.

16. Costa E, Sacramento E, Lopes AA, Carlos Bina J. Facial nerve palsy associated with leptospirosis. Revista da Sociedade Brasileira de Medicine Tropical. 2001:34(2):219-20.

17. Morgan M, Nathwani D. Facial palsy and infection: the unfolding story. Clin Infect Dis. 1992:14(1):263-71.

\section{Submit your next manuscript to BioMed Central and take full advantage of:}

- Convenient online submission

- Thorough peer review

- No space constraints or color figure charges

- Immediate publication on acceptance

- Inclusion in PubMed, CAS, Scopus and Google Scholar

- Research which is freely available for redistribution

Submit your manuscript at www.biomedcentral.com/submit 